\title{
Effect of impurities on collisional zonal-flow damping in tokamaks
}

\author{
S. Braun ${ }^{1}$, P. Helander ${ }^{1}$, E.A. Belli ${ }^{2}$ and J. Candy ${ }^{2}$ \\ 1 Max-Planck-Institut für Plasmaphysik, Teilinstitut Greifswald, \\ EURATOM-Association, D-17491 Greifswald, Germany \\ ${ }^{2}$ General Atomics, P.O. Box 85608, San Diego, CA 92186-5608, USA
}

March 13, 2009

\begin{abstract}
It is shown that the presence of highly charged impurity ions strongly increases the collisional damping of zonal flows in a low-collisionality tokamak plasma. A formula for the zonal-flow damping time is derived, which indicates that the damping is enhanced approximately by a factor $Z_{\text {eff }}$. If the aspect ratio is large, the enhancement is significantly larger. The zonal-flow damping time is also the time which the poloidal plasma rotation needs to respond to a sudden change in the pressure gradients.

PACS numbers: 52.25.Dg, 52.25.Vy, 52.55.Fa
\end{abstract}

\section{Introduction}

In gyrofluid [1, 2] and gyrokinetic [3] turbulence simulations, it is observed that so-called zonal flows strongly affect the rate at which turbulent fluctuations cause plasma transport across the magnetic field $[4,5]$. Zonal flows are bands of poloidal and toroidal rotation with relatively short radial wave-length. They are seen to reduce the turbulent transport by "shearing apart" the turbulent eddies, thus reducing their radial extent and suppressing the radial flux they produce. Zonal flows are driven by turbulent Reynolds stress and are damped by various mechanisms, including Coulomb collisions between the circulating ions, which are free to rotate around the torus and thus contribute to the parallel zonal flow, and the trapped ones, which are locked in their orbits on the outboard side of the torus.

In this paper we extend the analysis of earlier papers by Morries, Haines and Hastie [6], Hinton and Rosenbluth [7], and Xiao, Catto and Molvig [8], where the response of a tokamak plasma to an imposed radial electric field was calculated. At $t=0$ a short-wavelength radial electric field is imposed as an initial condition, and one asks how the plasma responds on time scales longer than the ion bounce time. If the plasma is in the "banana" regime of small collisionality, 
so that the collision time exceeds the bounce time, the evolution occurs in two separate stages: a relatively fast collisionless stage followed by a longer stage where ion-ion collisions are important. Both the collisionless and the collisional responses were calculated in Refs. [7, 8], and the result was expressed in terms of plasma polarisation. Because the ion orbits can move radially to some extent, the plasma is polarisable and tends to shield out (incompletely) the externally imposed radial electric field. The question is when and to which extent this happens, i.e., how the radial electric field evolves in time.

The primary purpose of the present paper is to establish how the presence of heavy ion impurities affects this evolution. We consider a plasma consisting of three species: hydrogenic bulk ions, electrons, and highly charged impurity ions. One would expect that the latter affect the collisional response of the ions, and we calculate in detail how this happens. As shown in Sec. 2 below, the appropriate equation governing zonal evolution is derived from the requirement that angular momentum should be conserved. When the radial electric field is first applied, the plasma quickly (i.e., on the ion cyclotron time scale) acquires an $E \times B$ drift perpendicular to the magnetic field. On the longer time scale of bounce motion, the plasma starts moving in the parallel direction, and this motion is modified on the yet longer time scale of ion-ion collisions, which compel the plasma ions to be Maxwellian as $t \rightarrow \infty$. Whilst the ion distribution function evolves in accordance with the drift kinetic equation, the radial electric field adjusts to keep the angular momentum constant. If $Z_{\text {eff }}-1=\mathcal{O}(1)$ and the impurities are highly charged, they contribute little to the total angular momentum but significantly increase the bulk ion collision frequency. Then the collisionless response of the plasma (and the so-called Rosenbluth-Hinton residual [9]) is not affected, but the collisional zonal flow damping is enhanced. In Sec. 3 below, we find that the time history of this damping can be calculated by the eigenfunction expansion of the collision operator introduced in Ref. [10]. Interestingly however, we also find, in Sec. 3.1, that if one is merely interested in calculating the overall damping time of the radial electric field $E_{r}(t)$,

$$
\tau_{p}=\int_{0}^{\infty}\left(\frac{E_{r}(t)}{E_{r}(\infty)}-1\right) d t
$$

rather than the entire time history, then the problem can be reduced to an equation routinely solved in neoclassical transport theory. This equation can also be solved using a more sophisticated collision operator. In Sec. 3.2, we compare our analytical results for the damping time with the numerical results of the NEO code [11] for different collision operators. We find numerics and analytics to be in good agreement when the same collision operator is used, and furthermore, the simulation indicates that the simple operator used in the analytical calculation seems to be a sufficiently accurate approximation. A discussion about the applicability of the presented calculations is given in Sec. 4, and our conclusions are summarised in Sec. 5 . 


\section{Basic equations}

\subsection{Kinetic equation}

For a plasma consisting of hydrogenic bulk ions and a single species of highly charged impurities with charge $q_{z}=Z q_{i}=Z e, Z \gg 1$, and

$$
\frac{Z^{2} n_{z}}{n_{i}} \sim \frac{Z m_{i}}{m_{z}} \sim 1
$$

we consider the drift kinetic equation corresponding to each particle species,

$$
\frac{\partial f_{a}}{\partial t}+\left(\boldsymbol{v}_{\|}+\boldsymbol{v}_{\boldsymbol{d}}\right) \cdot \nabla f_{a}+\dot{w} \frac{\partial f_{a}}{\partial w}=C_{a}\left(f_{a}\right)
$$

where $\boldsymbol{v}_{d}$ is the drift velocity, $w=\frac{1}{2} m_{a} v^{2}$ the kinetic energy and $\dot{w}=-e_{a}\left(\boldsymbol{v}_{\|}+\right.$ $\left.\boldsymbol{v}_{\boldsymbol{d}}\right) \cdot \boldsymbol{\nabla} \phi$ represents a drive in the form of a given electrostatic zonal flow potential $\phi(\psi, t)$. Both species are assumed to be in the banana regime, which requires the usual banana regime assumption of small effective collision frequency, $\left(\nu_{D}^{z z} q R\right) /\left(\epsilon^{3 / 2} v_{t h_{z}}\right) \ll 1$. Here $\nu_{D}^{z z}$ is the impurity collision frequency, $q$ the safety factor, $R$ the major radius and $v_{t h_{z}}$ the impurity thermal velocity. $\epsilon \equiv r / R_{0}$ is the inverse aspect ratio with minor radius $r$ and the on-axis value of the major radius $R_{0}$. Furthermore, $\boldsymbol{B}=I(\psi) \nabla \varphi+\nabla \varphi \times \nabla \psi$. Expanding in $\delta_{a}=\frac{\rho_{a}}{L} \ll 1$, where $\rho_{a}$ denotes the Larmor radius of the species $a$, one finds the zeroth order distribution function to be a Maxwellian; the remaining first order equation then reads

$$
\frac{\partial f_{a_{1}}}{\partial t}+v_{\|} \nabla_{\|}\left(f_{a_{1}}+\frac{I v_{\|}}{\Omega_{a}} \frac{e_{a} \phi^{\prime}}{T_{a}} f_{a_{0}}\right)=C\left(f_{a_{1}}\right) .
$$

Here we have neglected $\boldsymbol{v}_{d} \cdot \boldsymbol{\nabla} f_{a_{0}}$ as this term causes ordinary neoclassical transport, which adds linearly to the effects we want to study, and rewritten $\dot{w}$ in terms of the parallel gradient

$$
\dot{w} \frac{\partial f_{a_{0}}}{\partial w}=v_{\|} \nabla_{\|}\left(\frac{I v_{\|}}{\Omega_{a}}\right) \frac{e_{a} \phi^{\prime}}{T_{a}} f_{a_{0}}
$$

where a prime shall always denote derivation with respect to $\psi \cdot \Omega_{a}=e_{a} B / m_{a}$ denotes the gyro-frequency of the corresponding species. This notation suggests splitting off the adiabatic part from the distribution function by letting

$$
f_{a_{1}}=g_{a}-\frac{I v_{\|}}{\Omega_{a}} \frac{e_{a} \phi^{\prime}}{T_{a}} f_{a_{0}}
$$

If we assume the distribution function to vary on time scales much longer than the bounce time $\tau_{b}$ and the collision frequency $\nu$ to be much smaller than the bounce frequency $\omega_{b}$, we can further expand $g_{a}$ to find in zeroth order

$$
v_{\|} \nabla_{\|} g_{a_{0}}=0,
$$


and in first order

$$
\frac{\partial g_{a_{0}}}{\partial t}-\frac{I v_{\|}}{\Omega_{a}} \frac{e_{a}}{T_{a}} \frac{\partial \phi^{\prime}}{\partial t} f_{a_{0}}+v_{\|} \nabla_{\|} g_{a_{1}}=C\left(f_{a_{1}}\right) .
$$

Note that, due to symmetry arguments, $g_{a_{0}}$ vanishes in the trapped region of velocity space for any particle species [12] and we therefore only have to consider passing particles, i.e. particles with $0 \leq \lambda \leq \lambda_{c} \equiv B_{0} / B_{\max }$ where the pitch angle variable $\lambda$ is defined as $\lambda=v_{\perp}^{2} B_{0} / v^{2} B$. Furthermore, we define $B_{0}$ via $B_{0}^{2}=\left\langle B^{2}\right\rangle$, where angular brackets denote the flux-surface average,

$$
\langle Q(\psi)\rangle \equiv \oint \frac{Q(\psi, \theta)}{\boldsymbol{B} \cdot \boldsymbol{\nabla} \theta} d \theta / \oint \frac{d \theta}{\boldsymbol{B} \cdot \boldsymbol{\nabla} \theta}
$$

Multiplying (4) by $\frac{B}{\xi}, \xi \equiv \sigma v_{\|} / v$ where $\sigma=v_{\|} /\left|v_{\|}\right|$, and taking the flux surface average annihilates the parallel gradient, so that the differential equation we need to solve becomes

$$
\left\langle\frac{B}{\xi}\left(\frac{\partial g_{a_{0}}}{\partial t}-C\left(f_{a_{1}}\right)\right)\right\rangle=\frac{m_{a} I}{T_{a}} \sigma v f_{a_{0}} \frac{\partial \phi^{\prime}}{\partial t},
$$

together with the boundary condition $g_{a_{0}}\left(\lambda_{c}\right)=0$ needed for continuity of the distribution function at the trapped-passing boundary. This equation will be solved in section 3 for both bulk ions and impurities, taking into account the different collisional behaviour of the two species.

\subsection{Neoclassical polarisation}

In order to study the response of the plasma to a zonal flow potential we couple the kinetic equation of the previous section with the gyrokinetic quasineutrality condition in the limit of small ion gyroradius $\left(k_{\perp} \rho_{i} \ll 1\right)$,

$$
e n_{e}=\sum_{a=i, z} q_{a}\left(N_{a}+\nabla \cdot\left(\frac{n_{a}}{\Omega_{a} B} \nabla \phi\right)\right),
$$

where $n_{a}$ denotes the number density for the respective species and $N_{a}$ the guiding centre density which is defined by $N_{a}\left(\boldsymbol{R}_{a}\right)=\left.\int f\left(\boldsymbol{R}_{a}, \boldsymbol{v}, t\right) d^{3} v\right|_{\boldsymbol{R}_{a} \text { fixed }}$ where $\boldsymbol{R}_{a}=\boldsymbol{r}-\boldsymbol{b} \times \boldsymbol{v} / \Omega_{a}$ is the guiding-centre position and $\boldsymbol{b}$ the unit vector along the magnetic field. Since the electrons are frozen into the field, the electron density is conserved and we get

$$
\frac{\partial}{\partial t}\left[N_{i}+Z N_{z}+\nabla \cdot\left(\frac{\left(m_{i} n_{i}+m_{z} n_{z}\right) \nabla \phi}{e B^{2}}\right)\right]=0 .
$$

Taking the flux surface average and using the relation

$$
\langle\boldsymbol{\nabla} \cdot \boldsymbol{A}\rangle=\frac{1}{V^{\prime}} \frac{\partial}{\partial \psi} V^{\prime}\langle\boldsymbol{A} \cdot \nabla \psi\rangle
$$


which holds for any vector $\boldsymbol{A}$ and where $V(\psi)$ is the volume within the flux surface $\psi,(6)$ can be rewritten as

$$
\begin{aligned}
& \frac{\partial}{\partial t}\left\langle\frac{\left(m_{i} n_{i}+m_{z} n_{z}\right)|\nabla \psi|^{2}}{B^{2}} \phi^{\prime}\right. \\
& \left.-\int \frac{I v_{\|}}{B}\left(m_{i} g_{i_{0}}+m_{z} g_{z_{0}}-\frac{I v_{\|}}{B} \phi^{\prime}\left(\frac{m_{i}^{2}}{T_{i}} f_{i_{0}}+\frac{m_{z}^{2}}{T_{z}} f_{z_{0}}\right)\right) d^{3} v\right\rangle=0,
\end{aligned}
$$

where we have used

$$
\begin{aligned}
\frac{\partial\left\langle N_{a}\right\rangle}{\partial t} & =-\frac{1}{V^{\prime}} \frac{\partial}{\partial \psi} V^{\prime}\left\langle\int f_{a_{1}} \boldsymbol{v}_{d} \cdot \nabla \psi d^{3} v\right\rangle \\
& =-\frac{1}{V^{\prime}} \frac{\partial}{\partial \psi} V^{\prime}\left\langle\int g_{a} v_{\|} \nabla_{\|}\left(\frac{I v_{\|}}{\Omega_{a}}\right) d^{3} v\right\rangle \\
& =\frac{1}{V^{\prime}} \frac{\partial}{\partial \psi} V^{\prime}\left\langle\int \frac{I v_{\|}^{2}}{\Omega_{a}} \nabla_{\|} g_{a} d^{3} v\right\rangle \\
& =-\frac{1}{V^{\prime}} \frac{\partial}{\partial \psi} V^{\prime}\left\langle\int \frac{I v_{\|}}{\Omega_{a}}\left(\frac{\partial g_{a_{0}}}{\partial t}-\frac{I v_{\|}}{\Omega_{a}} \frac{e_{a}}{T_{a}} \frac{\partial \phi^{\prime}}{\partial t} f_{a_{0}}-C_{a}\left(f_{a_{1}}\right)\right) d^{3} v\right\rangle .
\end{aligned}
$$

Note that the terms containing the collision operator drop out due to momentum conservation. Noticing that

$$
\left\langle\frac{I}{B} \int m_{a} v_{\|} f_{a_{1}} d^{3} v\right\rangle=\left\langle m_{a} n_{a} R V_{a_{\|}} \boldsymbol{b} \cdot \boldsymbol{e}_{\boldsymbol{\varphi}}\right\rangle=L_{\|}
$$

is the angular momentum of the parallel motion while that of the perpendicular $E \times B$ motion is

$$
L_{\perp}=\left\langle m_{a} n_{a} \frac{\boldsymbol{b} \times \boldsymbol{\nabla} \phi}{B} \cdot R \boldsymbol{e}_{\boldsymbol{\varphi}}\right\rangle=-m_{a} n_{a}\left\langle\frac{|\boldsymbol{\nabla} \psi|^{2}}{B^{2}}\right\rangle \phi^{\prime},
$$

we see that (7) represents the conservation of toroidal angular momentum. Here $V_{a_{\|}}$denotes the parallel velocity of the particle species $a, \boldsymbol{b}$ is the unit vector along the magnetic field and $\boldsymbol{e}_{\boldsymbol{\varphi}}$ the unit vector in the $\varphi$ direction.

For later calculations, it is convenient to Laplace transform the equations we are dealing with and choose the initial conditions such that there is no initial parallel rotation of the plasma, i.e. $f_{i_{1}}(0)=f_{z_{1}}(0)=0$. Defining the neoclassical polarisation $\hat{P}$ as

$$
\hat{P}=\sum_{a=i, z}\left\langle\frac{I}{B} \int m_{a} v_{\|} \hat{g}_{a_{0}} d^{3} v\right\rangle / \sum_{a=i, z}\left\langle m_{a} n_{a} R^{2}\right\rangle \hat{\phi}^{\prime},
$$

we can represent the potential response as

$$
\hat{\phi}^{\prime}(p)=\frac{1}{p} \phi^{\prime}(0) \frac{\left\langle\frac{|\nabla \psi|^{2}}{B^{2}}\right\rangle}{\left\langle R^{2}\right\rangle(1-\hat{P})} .
$$


Laplace transformed quantities are denoted by a hat; note that we have anticipated $\hat{g}_{a_{0}}(p)$ to be proportional to $\hat{\phi}^{\prime}(p)$ and taken the initial condition on the distribution function to be $f_{a_{1}}(t=0)=0$. For the limit of a collisionless, large-aspect ratio tokamak with circular flux surfaces, we find, in agreement with Hinton and Rosenbluth [7], the potential response to be

$$
\begin{aligned}
\hat{\phi}^{\prime}(p) & =\frac{\sum_{a}\left\langle\frac{m_{a} n_{a}|\nabla \psi|^{2}}{B^{2}}\right\rangle}{\sum_{a}\left\langle m_{a} n_{a} R^{2}\right\rangle(1-\hat{P})} \frac{\phi^{\prime}(0)}{p} \\
& \approx \frac{\sum_{a} m_{a} n_{a} R^{2} \frac{B_{p}^{2}}{B^{2}}}{\sum_{a} m_{a} n_{a} \frac{r^{2}}{q^{2}}\left(1+1.64 \frac{q^{2}}{\sqrt{\epsilon}}\right)} \frac{\phi^{\prime}(0)}{p} \\
& =\frac{1}{1+1.64 \frac{q^{2}}{\sqrt{\epsilon}}} \frac{\phi^{\prime}(0)}{p},
\end{aligned}
$$

where $q$ is the safety factor and $B_{p}$ the poloidal magnetic field strength. Here we have used that the collisionless solution of (5) is

$$
g_{a_{0}}=\frac{m_{a} I \phi^{\prime}}{T_{a}}\left\langle\frac{B}{v_{\|}}\right\rangle^{-1} f_{a_{0}}
$$

and the value of $\left\langle B / v_{\|}\right\rangle^{-1}$ can be computed numerically. This is the same result as for a pure plasma. When collisions are included, one can consider the limit of very late times $(p \rightarrow 0)$ and determine the influence of impurity ions on that residual. As we will find later on, the polarisation $\hat{P}$ vanishes in this limit, regardless of whether impurities are present or not, thus the residual remains unaffected and equals

$$
\hat{\phi}^{\prime}(p \rightarrow 0) \approx \frac{\epsilon^{2}}{q^{2}} \frac{\phi^{\prime}(0)}{p} .
$$

Our main task is now to calculate the polarisation $\hat{P}$ when collisions are included, which requires calculating the distribution functions of all different particle species.

\section{Potential response}

Using the results of the previous sections, we now solve Eq. (5) for the impurities. We start from

$$
\left\langle\frac{B}{\xi}\right\rangle \frac{\partial g_{z_{0}}}{\partial t}-\nu_{D}^{z z}\left\langle\frac{B}{\xi} \mathscr{L}\left(f_{z_{1}}\right)\right\rangle=\frac{m_{z}}{T_{z}} v \sigma f_{z_{0}}\left(I \frac{\partial \phi^{\prime}}{\partial t}+\nu_{D}^{z z}\left\langle B u_{z_{\|}}\right\rangle\right),
$$

where we have used the Connor model [13] for the collision operator,

$$
C_{z z}\left(f_{z_{1}}\right)=\nu_{D}^{z z}\left(\mathscr{L}\left(f_{z_{1}}\right)+\frac{m_{z} v_{\|} u_{z_{\|}}}{T_{z}} f_{z_{0}}\right),
$$


denoting the Lorentz scattering operator, and the quantity $u_{z_{\|}}$can be calculated from the condition of momentum conservation in self-collisions. For two arbitrary species,

$$
\begin{aligned}
\nu_{D}^{a b} & =\hat{\nu}_{a b} \frac{\Phi\left(x_{b}\right)-G\left(x_{b}\right)}{x_{a}^{3}} \equiv \hat{\nu}_{a b} \frac{H\left(x_{b}\right)}{x_{a}^{3}} \\
\hat{\nu}_{a b} & =\frac{n_{b} e_{a}^{2} e_{b}^{2} \ln \Lambda}{4 \pi \epsilon_{0}^{2} m_{a}^{2} v_{t h_{a}}^{3}}
\end{aligned}
$$

and $x_{a}=v / v_{t h_{a}}$ where $v_{t h_{a}}=\left(2 T_{a} / m_{a}\right)^{1 / 2}$ is the thermal velocity and $\ln \Lambda$ the Coulomb logarithm. $\Phi$ denotes the error function and $G$ the Chandrasekhar function, defined as

$$
G(x)=\frac{\Phi(x)-x \phi^{\prime}(x)}{2 x^{2}}
$$

The index $z z$ shall denote self-collisions between the impurities; impurity-ion collisions can be neglected since the effect of impurity-ion collisions is smaller than that of self-collisions by a factor of the square root of the mass ratio.

In order to solve Eq. (11), we use an eigenfunction technique [8, 10] and expand the distribution function in eigenfunctions $\Lambda_{n}(\psi, \lambda)$, determined by the eigenvalue problem

$$
\left\langle\frac{B}{\xi} \mathscr{L}\left(\Lambda_{n}\right)\right\rangle=2 B_{0} \frac{\partial}{\partial \lambda} \lambda\langle\xi\rangle \frac{\partial \Lambda_{n}}{\partial \lambda}=-\chi_{n}\left\langle\frac{B}{\xi}\right\rangle \Lambda_{n}
$$

in the domain $0<\lambda<\lambda_{c}$. The boundary conditions are chosen such that the transition at the trapped-passing boundary is continuous, and thus $\Lambda_{n}\left(\lambda=\lambda_{c}\right)$ $=0$. Note that the equation has a regular singular point at $\lambda=0$ and we therefore demand $\Lambda_{n}(\lambda=0)$ to stay finite as a natural boundary condition. As the Lorentz operator is self-adjoint and the weight $\langle B / \xi\rangle \geq 0$, this is a Sturm-Liouville problem. For convenience, we use the normalisation

$$
\int_{0}^{\lambda_{c}} \Lambda_{n} d \lambda=\frac{2}{3}
$$

Expanding $g_{i_{0}}$ and $g_{z_{0}}$ in an eigenfunction series as

$$
g_{a_{0}}=\sigma \sum_{n=1}^{\infty} b_{a n}(\psi, v) \Lambda_{n}(\psi, \lambda)
$$

and Laplace transforming Eq. (11), we get

$$
\begin{array}{r}
p \hat{b}_{z n}-b_{z n}\left(t \ll \tau_{a}\right)+\chi_{n} \nu_{D}^{z z} \hat{b}_{z n}=\beta_{n} \frac{m_{z}}{T_{z}} v f_{z_{0}}\left(I p \hat{\phi}^{\prime}-I \phi^{\prime}\left(t \ll \tau_{a}\right)\right. \\
\left.+I \hat{\phi}^{\prime} \nu_{D}^{z z}+\nu_{D}^{z z}\left\langle B \hat{u}_{z_{\|}}\right\rangle\right)
\end{array}
$$


where we define

$$
\beta_{n} \equiv \frac{B_{0} \int_{0}^{\lambda_{c}} \Lambda_{n} d \lambda}{\int_{0}^{\lambda_{c}}\left\langle\frac{B}{\xi}\right\rangle \Lambda_{n}^{2} d \lambda}=\frac{2 B_{0}}{3 \int_{0}^{\lambda_{c}}\left\langle\frac{B}{\xi}\right\rangle \Lambda_{n}^{2} d \lambda} .
$$

As $g_{a_{0}}$ changes rapidly on the bounce time scale but afterwards relaxes much more slowly on the collision time scale, we cannot use the initial condition $f_{a_{1}}=0$ at $t=0$ we used in section 2.2 for studying the effect on the slower time scale as this initial condition would violate the condition (3) due to our expansion in $\omega_{b}$. Therefore, we take the initial condition at $t \ll \tau_{a}$, which shall express a time later than a few bounce times but much earlier than the collision time, to be consistent with our ordering. This initial condition we use for $g_{a_{0}}$ can be obtained from the collisionless limit (10), which gives

$$
g_{a_{0}}\left(t \ll \tau_{a}\right)=\frac{m_{a}}{T_{a}} f_{a_{0}}\left\langle\frac{B}{v_{\|}}\right\rangle^{-1} I \phi^{\prime}\left(t \ll \tau_{a}\right),
$$

where $\tau_{a}$ denotes the collision time. Thus, the two terms in Eq. (13) containing the initial conditions cancel each other.

The distribution function $\hat{f}_{z_{1}}$ is then given by the following expression

$$
\hat{f}_{z_{1}}=\frac{m_{z}}{T_{z}} f_{z_{0}}\left[\sigma v \sum_{n=1}^{\infty} \frac{\beta_{n} \Lambda_{n}}{B_{0}\left(p+\chi_{n} \nu_{D}^{z z}\right)}\left(I \hat{\phi}^{\prime}\left(p+\nu_{D}^{z z}\right)+\nu_{D}^{z z}\left\langle B \hat{u}_{z_{\|}}\right\rangle\right)-\frac{I v_{\|}}{B} \hat{\phi}^{\prime}\right]
$$

To conserve momentum in like-particle collisions, we calculate $\hat{u}_{z_{\|}}$from

$$
\int v_{\|} C_{z z}\left(\hat{f}_{z}\right) d^{3} v \stackrel{!}{=} 0
$$

Exploiting the self-adjointness of the Lorentz operator and the relation $\mathscr{L}\left(v_{\|}\right)=-v_{\|}$, we arrive at the following expression [12]

$$
\hat{u}_{z_{\|}}=\frac{\int v_{\|} \nu_{D}^{z z} \hat{f}_{z_{1}} d^{3} v}{n_{z}\left\{\nu_{D}^{z z}\right\}}
$$

with the velocity-space average defined as

$$
\{F(v)\}_{a} \equiv \int F(v) \frac{m_{a} v_{\|}^{2}}{n_{a} T_{a}} f_{a_{0}} d^{3} v
$$

As we need to calculate the quantity $\left\langle B \hat{u}_{z_{\|}}\right\rangle$, we multiply by $B$ and take the flux surface average to find

$$
\left\langle B \hat{u}_{z_{\|}}\right\rangle=-I \hat{\phi}^{\prime}\left(1-\frac{\frac{p}{\left\{\nu_{D}^{z z}\right\}} \sum_{n=1}^{\infty} \beta_{n}\left\{\frac{\nu_{D}^{z z}}{p+\chi_{n} \nu_{D}^{z z}}\right\}}{1-\frac{1}{\left\{\nu_{D}^{z z}\right\}} \sum_{n=1}^{\infty} \beta_{n}\left\{\frac{\left(\nu_{D}^{z z}\right)^{2}}{p+\chi_{n} \nu_{D}^{z z}}\right\}}\right),
$$


which, in the long-time limit (small $p)$, simplifies to

$$
\left\langle B \hat{u}_{z_{\|}}\right\rangle=-I \hat{\phi}^{\prime}\left(1-p \tau_{0}+\mathcal{O}\left(p^{2}\right)\right)
$$

where we define

$$
\tau_{0}^{-1} \equiv\left\{\nu_{D}^{z z}\right\}\left(\left(\sum_{n=1}^{\infty} \frac{\beta_{n}}{\chi_{n}}\right)^{-1}-1\right) .
$$

We can show $\sum_{n=1}^{\infty} \beta_{n} / \chi_{n}$ to equal the "effective" fraction of circulating particles introduced in [14], and thus always to be smaller than 1 by the following argument: Consider the problem

$$
\left\langle\frac{B}{\xi} \mathscr{L}(h)\right\rangle=-B_{0}, \quad h\left(\lambda_{c}\right)=0, \quad h(0)<\infty .
$$

Inserting the expansion $h(\lambda)=\sum_{n=1}^{\infty} h_{n} \Lambda_{n}(\lambda)$ in (17) yields

$$
\sum_{n=1}^{\infty} \chi_{n} h_{n} \Lambda_{n}=B_{0}\left\langle\frac{B}{\xi}\right\rangle^{-1}=\sum_{n=1}^{\infty} \beta_{n} \Lambda_{n},
$$

and thus

$$
h(\lambda)=\sum_{n=1}^{\infty} \frac{\beta_{n}}{\chi_{n}} \Lambda_{n}
$$

Consequently,

$$
\int_{0}^{\lambda_{c}} h(\lambda) d \lambda=\sum_{n=1}^{\infty} \frac{\beta_{n}}{\chi_{n}} \int_{0}^{\lambda_{c}} \Lambda_{n} d \lambda=\frac{2}{3} \sum_{n=1}^{\infty} \frac{\beta_{n}}{\chi_{n}} .
$$

On the other hand, straight-forward integration of (17) leads to

$$
h(\lambda)=\frac{1}{2} \int_{\lambda}^{\lambda_{c}} \frac{d \lambda^{\prime}}{\left\langle\sqrt{1-\lambda^{\prime} \frac{B}{B_{0}}}\right\rangle},
$$

and we can combine both equations to yield

$$
\begin{aligned}
\sum_{n=1}^{\infty} \frac{\beta_{n}}{\chi_{n}} & =\frac{3}{2} \int_{0}^{\lambda_{c}} h(\lambda) d \lambda=\frac{3}{4} \int_{0}^{\lambda_{c}} \frac{\lambda d \lambda}{\left\langle\sqrt{1-\lambda \frac{B}{B_{0}}}\right\rangle} \\
& \equiv f_{c}
\end{aligned}
$$

where $f_{c}$ is the "effective" fraction of circulating particles. This quantity is always smaller than or equal to 1 , with equality if and only if $B=B_{\max }=$ const, 
which is not the case in a toroidal device. Thus, $\tau_{0}$ and therefore the momentum restoring term $\left\langle B \hat{u}_{i_{\|}}\right\rangle$are well-defined. For a large aspect ratio tokamak with circular flux surfaces, $f_{c} \cong 1-1.46 \sqrt{\epsilon}$.

Having derived the distribution function for the impurities, we proceed to the slightly more complicated task of calculating that of the bulk ions. With the collision operator

$$
C_{i}\left(f_{i_{1}}\right)=\left(\nu_{D}^{i i}+\nu_{D}^{i z}\right) \mathscr{L}\left(f_{i_{1}}\right)+\frac{m_{i}}{T_{i}} v_{\|} f_{i_{0}}\left(\nu_{D}^{i i} u_{i_{\|}}+\nu_{D}^{i z} V_{z_{\|}}\right),
$$

where we again have used the Connor operator for self-collisions and the last term on the right-hand side represents ion-impurity collisions, the differential equation becomes

$$
\begin{aligned}
& \left\langle\frac{B}{\xi}\right\rangle \frac{\partial g_{i_{0}}}{\partial t}-\left(\nu_{D}^{i i}+\nu_{D}^{i z}\right)\left\langle\frac{B}{\xi} \mathscr{L}\left(f_{i 1}\right)\right\rangle \\
= & \frac{m_{i}}{T_{i}} v \sigma f_{i_{0}}\left(I \frac{\partial \phi^{\prime}}{\partial t}+\nu_{D}^{i i}\left\langle B u_{i_{\|}}\right\rangle+\nu_{D}^{i z}\left\langle B V_{z_{\|}}\right\rangle\right) .
\end{aligned}
$$

Following the same steps as in the calculation for the impurities, we arrive at

$$
\begin{aligned}
\hat{f}_{i 1}= & \frac{m_{i}}{T_{i}} f_{i_{0}}\left[\sigma v \sum _ { n = 1 } ^ { \infty } \frac { \beta _ { n } \Lambda _ { n } } { B _ { 0 } ( p + \chi _ { n } ( \nu _ { D } ^ { i i } + \nu _ { D } ^ { i z } ) ) } \left(I p \hat{\phi}^{\prime}+\nu_{D}^{i i}\left(I \hat{\phi}^{\prime}+\left\langle B \hat{u}_{i_{\|}}\right\rangle\right)\right.\right. \\
& \left.\left.+\nu_{D}^{i z}\left(I \hat{\phi}^{\prime}+\left\langle B V_{z_{\|}}\right\rangle\right)\right)-\frac{I v_{\|}}{B} \hat{\phi}^{\prime}\right] .
\end{aligned}
$$

The two terms on the right that remain to be calculated are the momentum restoring coefficient $u_{i}$ and additionally the term containing the impurity flow speed $V_{z_{\|}}$. As we need this second term for the calculation of $u_{i \|}$, we start by multiplying this term by $B / v_{\|}$and take the flux surface average to find

$$
\begin{aligned}
\left\langle B V_{z_{\|}}\right\rangle & =\left\langle\frac{B}{n_{z}} \int v_{\|} \hat{f}_{z_{1}} d^{3} v\right\rangle \\
& =\sum_{n=1}^{\infty} \beta_{n}\left\{\frac{p I \hat{\phi}^{\prime}+\nu_{D}^{z z}\left(I \hat{\phi}^{\prime}+\left\langle B \hat{u}_{z_{\|}}\right\rangle\right)}{p+\chi_{n} \nu_{D}^{z z}}\right\}-I \hat{\phi}^{\prime}
\end{aligned}
$$

which, in the long-time limit, becomes

$$
\begin{aligned}
\left\langle\frac{B}{n_{z}} \int v_{\|} \hat{f}_{z_{1}} d^{3} v\right\rangle & =-I \hat{\phi}^{\prime}\left(1-\sum_{n=1}^{\infty} \beta_{n} p\left\{\frac{1+\nu_{D}^{z z} \tau_{0}}{p+\chi_{n} \nu_{D}^{z z}}\right\}\right) \\
& =-I \hat{\phi}^{\prime}\left(1-p \tau_{1}+\mathcal{O}\left(p^{2}\right)\right)
\end{aligned}
$$

where

$$
\tau_{1} \equiv f_{c}\left(\left\{\frac{1}{\nu_{D}^{z z}}\right\}+\tau_{0}\right)
$$


and $\tau_{0}$ was already defined in (16). To calculate

$$
\hat{u}_{i_{\|}}=\frac{\int v_{\|} \nu_{D}^{i i} \hat{f}_{i_{1}} d^{3} v}{n_{i}\left\{\nu_{D}^{i i}\right\}}
$$

we insert the distribution function $\hat{f}_{i_{1}}$ and the impurity term calculated above, and obtain

$$
\left\langle B \hat{u}_{i_{\|}}\right\rangle=-I \hat{\phi}^{\prime}+\frac{\sum_{n=1}^{\infty} \beta_{n}\left(\left\{\nu_{D}^{i i} \frac{p I \hat{\phi}^{\prime}+\nu_{D}^{i z}\left(I \hat{\phi}^{\prime}+\left\langle B V_{z_{\|}}\right\rangle\right)}{p+\chi_{n}\left(\nu_{D}^{i 2}+\nu_{D}^{i z}\right)}\right\}\right)}{\left\{\nu_{D}^{i i}\right\}-\sum_{n=1}^{\infty} \beta_{n}\left\{\frac{\left(\nu_{D}^{i i}\right)^{2}}{p+\chi_{n}\left(\nu_{D}^{i 2}+\nu_{D}^{i z}\right)}\right\}}
$$

and in the approximation $p \tau_{i i} \ll 1$

$$
\left\langle B \hat{u}_{i_{\|}}\right\rangle=-I \hat{\phi}^{\prime}\left(1-p \tau_{2}+\mathcal{O}\left(p^{2}\right)\right)
$$

with

$$
\tau_{2}=\frac{f_{c}\left\{\frac{\nu_{D}^{i i}\left(1+\nu_{D}^{i z} \tau_{1}\right)}{\nu_{D}^{i 2}+\nu_{D}^{i z}}\right\}}{\left\{\nu_{D}^{i i}\right\}-f_{c}\left\{\frac{\left(\nu_{D}^{i i}\right)^{2}}{\nu_{D}^{i 2}+\nu_{D}^{i z}}\right\}}
$$

Note that, as

$$
\frac{1}{\left\{\nu_{D}^{i i}\right\}}\left\{\frac{\left(\nu_{D}^{i i}\right)^{2}}{\nu_{D}^{i i}+\nu_{D}^{i z}}\right\} \leq \frac{1}{\left\{\nu_{D}^{i i}\right\}}\left\{\frac{\left(\nu_{D}^{i i}\right)^{2}}{\nu_{D}^{i i}}\right\}=1,
$$

the denominator of this expression is larger than the fraction of trapped particles, i.e. non-zero, and the momentum restoring term is again well-defined. Before finally calculating the plasma polarisation, we note that the impurity and bulk ion distribution functions, Eqs. (14) and (19), respectively, adjust to the radial electric field on very different time scales. The impurity ions adjust on the time scale $\tau_{0} \sim \tau_{1} \sim 1 / \nu_{D}^{z z}$ but the bulk ions do so only on the time scale $\tau_{2} \sim 1 / \nu_{D}^{i i}$, which is a factor $Z^{3 / 2}\left(Z_{\text {eff }}-1\right) \gg 1$ longer. Exploiting this property and the fact that $m_{z} n_{z} \sim \frac{1}{Z} m_{i} n_{i}$ in the large- $Z$ approximation, we find that

$$
\left\langle\int \frac{I v_{\|}}{B}\left(m_{i} \hat{g}_{i_{0}}+m_{z} \hat{g}_{z_{0}}\right) d^{3} v\right\rangle=\left\langle\frac{I}{B_{0}^{2}} \sum_{n=1}^{\infty} \beta_{n} m_{i} n_{i}\left\{\frac{p I \hat{\phi}^{\prime}\left(1+\tau_{2} \nu_{D}^{i i}\right)}{p+\chi_{n}\left(\nu_{D}^{i i}+\nu_{D}^{i z}\right)}\right\}\right\rangle,
$$

which can, in the long-time limit, be simplified to yield

$$
\left\langle\int \frac{I v_{\|}}{B}\left(m_{i} \hat{g}_{i_{0}}+m_{z} \hat{g}_{z_{0}}\right) d^{3} v\right\rangle=\left\langle\frac{I^{2}}{B_{0}^{2}} p \hat{\phi}^{\prime} f_{c} m_{i} n_{i}\left\{\frac{1+\tau_{2} \nu_{D}^{i i}}{\nu_{D}^{i i}+\nu_{D}^{i z}}\right\}\right\rangle .
$$

Thus, in leading order, the expression for the polarisation $\hat{P}$ becomes

$$
\hat{P}=\frac{I^{2}}{\left\langle R^{2}\right\rangle B_{0}^{2}} f_{c} p\left(\left\{\frac{1}{\nu_{D}^{i i}+\nu_{D}^{i z}}\right\}+\frac{f_{c}\left\{\frac{\nu_{D}^{i i}}{\nu_{D}^{i i}+\nu_{D}^{i z}}\right\}^{2}}{\left\{\nu_{D}^{i i}\right\}-f_{c}\left\{\frac{\left(\nu_{D}^{i i}\right)^{2}}{\nu_{D}^{i \nu}+\nu_{D}^{i z}}\right\}}\right)+\mathcal{O}\left(Z^{-1 / 2}\right)+\mathcal{O}\left(p^{2}\right) .
$$

In a large-aspect-ratio equilibrium, $I^{2} /\left(R^{2} B_{0}^{2}\right) \approx 1$. 


\subsection{Long-time limit}

The results from the previous section show that the detailed geometry of the flux surfaces matters little to the zonal flow response in the long-time limit (small $p$ ), since the only information from the eigenfunction expansion that survives this limit is the quantity $f_{c}=\sum_{n=1}^{\infty} \beta_{n} / \chi_{n}$. This suggests that the response on time scales longer than the bulk ion collision time can be obtained more easily. To this end, we reconsider Eq. (4) but neglect the small term containing the time derivative (in this limit, $\partial g_{a} / \partial t \sim p g_{a} \ll \nu_{D}^{a a} g_{a}$ ). Thus, instead of having to solve a partial differential equation depending on time, we arrive at the much simpler equation

$$
v_{\|} \nabla_{\|} g_{a}-\frac{e_{a} E_{a} v_{\|}}{T_{a}} f_{a_{0}}=C_{a}\left(f_{a}\right)
$$

with

$$
E_{a}=\frac{I}{\Omega_{a}} \frac{\partial \phi^{\prime}}{\partial t}
$$

regarded as given. This resembles a neoclassical Spitzer problem, except that the "electric field" $E_{a}$ is different for different species, and we can solve straightforwardly for both impurities and ions to find

$$
\hat{g}_{z_{0}}=\frac{1}{2 B_{0}} \frac{m_{z}}{T_{z}} \sigma v f_{z_{0}}\left(I \hat{\phi}^{\prime}\left(1+\frac{p}{\nu_{D}^{z z}}\right)+\left\langle B \hat{u}_{z_{\|}}\right\rangle\right) \int_{\lambda}^{\lambda_{c}} \frac{d \lambda^{\prime}}{\left\langle\sqrt{1-\lambda^{\prime} \frac{B}{B_{0}}}\right\rangle}+\mathcal{O}\left(p^{2}\right)
$$

and

$$
\begin{aligned}
\hat{g}_{i_{0}}= & \frac{1}{2 B_{0}} \frac{m_{i}}{T_{i}} \sigma v f_{i_{0}}\left(I \hat{\phi}^{\prime} \frac{p}{\nu_{D}^{i i}+\nu_{D}^{i z}}+\left(I \hat{\phi}^{\prime}+\left\langle B \hat{u}_{i_{\|}}\right\rangle\right) \frac{\nu_{D}^{i i}}{\nu_{D}^{i i}+\nu_{D}^{i z}}\right. \\
& \left.+\left(I \hat{\phi}^{\prime}+\left\langle B V_{z_{\|}}\right\rangle\right) \frac{\nu_{D}^{i z}}{\nu_{D}^{i i}+\nu_{D}^{i z}}\right) \int_{\lambda}^{\lambda_{c}} \frac{d \lambda^{\prime}}{\left\langle\sqrt{1-\lambda^{\prime} \frac{B}{B_{0}}}\right\rangle}+\mathcal{O}\left(p^{2}\right) .
\end{aligned}
$$

Thus, we arrive at

$$
\begin{aligned}
\hat{P}= & \frac{I^{2}}{\left\langle R^{2}\right\rangle B_{0}^{2}} f_{c} p\left(\left\{\frac{1}{\nu_{D}^{i i}+\nu_{D}^{i z}}\right\}+\frac{f_{c}\left\{\frac{\nu_{D}^{i i}}{\nu_{D}^{i i}+\nu_{D}^{i z}}\right\}^{2}}{\left\{\nu_{D}^{i i}\right\}-f_{c}\left\{\frac{\left(\nu_{D}^{i i}\right)^{2}}{\nu_{D}^{i i}+\nu_{D}^{i z}}\right\}}\right) \\
& +\mathcal{O}\left(Z^{-1 / 2}\right)+\mathcal{O}\left(p^{2}\right),
\end{aligned}
$$

which is the same result as with the eigenfunction expansion method.

As the inverse Laplace transform ranges over the whole $p$-space, we cannot formally transform the expression for the long-time limit back to $t$-space. 
Nonetheless, we can extract important information about the zonal flow damping time by considering how the potential approaches its final value

$$
\lim _{t \rightarrow \infty} \phi^{\prime}(t)=\frac{\left\langle\frac{|\nabla \psi|^{2}}{B^{2}}\right\rangle}{\left\langle R^{2}\right\rangle} \phi^{\prime}(0) \equiv \phi_{\infty}^{\prime}
$$

A suitable measure for the time scale on which $\phi^{\prime}(t)$ approaches $\phi_{\infty}^{\prime}$ is

$$
\tau_{p} \equiv \int_{0}^{\infty} \frac{\phi^{\prime}(t)-\phi_{\infty}^{\prime}}{\phi_{\infty}^{\prime}} d t .
$$

Using (9), we find

$$
\begin{aligned}
\tau_{p} & =\lim _{p \rightarrow 0} \int_{0}^{\infty} \frac{\phi^{\prime}(t)-\phi_{\infty}^{\prime}}{\phi_{\infty}^{\prime}} e^{-p t} d t \\
& =\frac{d \hat{P}}{d p}(0) .
\end{aligned}
$$

The effective zonal-flow damping time, $\tau_{p}$, thus corresponds exactly to the term, linear in $p$, that we have calculated explicitly in the polarisation (24). This expression is a simple function of $f_{c}$ but depends in a complicated way on $Z_{\text {eff }}$, as it involves velocity-space averages $\{\ldots\}$ of $\nu_{D}^{i i}$ and $\nu_{D}^{i z}$. In order to further simplify Eq. (24), we consider the limits of $Z_{\mathrm{eff}} \rightarrow 1$ and $Z_{\mathrm{eff}} \rightarrow \infty$ : In a pure plasma, the damping time becomes

$$
\left.\tau_{p}\right|_{\text {eff }}=1=\frac{I^{2}}{\left\langle R^{2}\right\rangle B_{0}^{2}} \frac{f_{c}}{\hat{\nu}_{i e}}\left(\left\{\frac{x^{3}}{H(x)}\right\}+\frac{f_{c}}{1-f_{c}} \frac{1}{\left\{\frac{H(x)}{x^{3}}\right\}}\right),
$$

whereas for $Z_{\mathrm{eff}} \rightarrow \infty$

$$
\left.\tau_{p}\right|_{Z_{\mathrm{eff}} \rightarrow \infty} \approx \frac{I^{2}}{\left\langle R^{2}\right\rangle B_{0}^{2}} f_{c} \frac{\left\{x^{3}\right\}}{\hat{\nu}_{i e}} \frac{1}{Z_{\mathrm{eff}}},
$$

where we used $\hat{\nu}_{i e} \approx \hat{\nu}_{i i}$ for large $Z$ and [12]

$$
\begin{aligned}
\nu_{D}^{i z} & \approx \frac{\hat{\nu}_{i z}}{x^{3}}=\frac{Z^{2} n_{z}}{n_{i}} \frac{\hat{\nu}_{i i}}{x^{3}}=\frac{Z_{\mathrm{eff}}-1}{1-\frac{1}{Z} Z_{\mathrm{eff}}} \frac{\hat{\nu}_{i i}}{x^{3}} \\
& \approx\left(Z_{\mathrm{eff}}-1\right) \frac{\hat{\nu}_{i e}}{x^{3}}
\end{aligned}
$$

where $Z_{\text {eff }}=\sum_{j}\left(Z_{j}^{2} n_{j}\right) / \sum_{j}\left(Z_{j} n_{j}\right)$ and the sum is taken over all ion species $j$. The normalisation to $\hat{\nu}_{i e}$ was chosen to account for the electron density being the quantity typically measured in experiment, and, furthermore, this normalisation is also used for the numerical simulations in the next chapter in order to avoid 


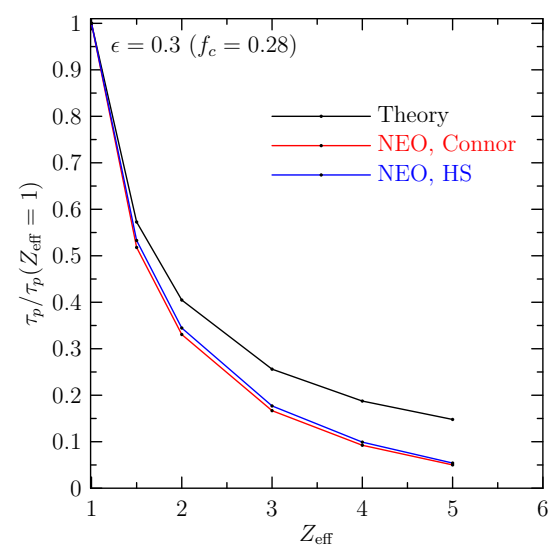

Figure 1: Normalised damping time versus effective charge

singular behaviour when $Z$ is close to $Z_{\text {eff }}$, as it would occur for normalising with respect to $\hat{\nu}_{i i}$ while keeping $n_{e}, Z$ and $Z_{\text {eff }}$ fixed. With the help of these values, we can construct the following interpolation formula for the damping time

$$
\begin{aligned}
\tau_{p} & =\frac{I^{2}}{\left\langle R^{2}\right\rangle B_{0}^{2}} \frac{f_{c}}{\hat{\nu}_{i e}}\left[\frac{\left\{x^{3}\right\}}{Z_{\mathrm{eff}}}+\left(\left\{\frac{x^{3}}{H(x)}\right\}+\frac{f_{c}}{1-f_{c}} \frac{1}{\left\{\frac{H(x)}{x^{3}}\right\}}-\left\{x^{3}\right\}\right) \frac{1}{Z_{\mathrm{eff}}^{4}}\right] \\
& \approx \frac{I^{2}}{\left\langle R^{2}\right\rangle B_{0}^{2}} \frac{f_{c}}{\hat{\nu}_{i e}}\left[\frac{4.51}{Z_{\mathrm{eff}}}+\left(0.87+2.49 \frac{f_{c}}{1-f_{c}}\right) \frac{1}{Z_{\mathrm{eff}}^{4}}\right],
\end{aligned}
$$

which is exact in the limits and sufficiently accurate for intermediate values of $Z_{\text {eff }}$.

\subsection{Comparison with numerical simulation}

Since the calculation of $\tau_{p}$ only requires solving a standard equation from neoclassical transport theory, a number of existing codes can be used for this purpose. In this section, we compare our analytical results with the results of the NEO code [11]. In standard form, NEO solves the multi-species, steady-state first-order drift-kinetic equation using an Eulerian numerical scheme. Here we have modified NEO to solve the Laplace-transformed Eq. (23) for $f_{a 1}$, i.e. by setting the usual neoclassical driver source term to zero in the drift-kinetic equation and adding the term $-I\left(e_{a} / T_{a}\right) v_{\|} f_{0 a} \hat{\phi}^{\prime}\left[-p / \Omega_{a}+\nabla_{\|}\left(v_{\|} / \Omega_{a}\right)\right]$ as the new right-hand side source term. Given as input $\hat{\phi}^{\prime}$ and $p$, the discretised kinetic equation is solved as a matrix problem for $f_{a 1}$ and the polarisation $\hat{P}$ is computed from Eq. (8). For these simulations, we model a deuterium species and a carbon species in a tokamak plasma with s- $\alpha$ geometry and the parameters $\alpha=q^{2} R_{0} d \beta / d r=0, R_{0} / a=3, q=2, T_{0 i}=T_{0 c}$. (s- $\alpha$ geometry refers 


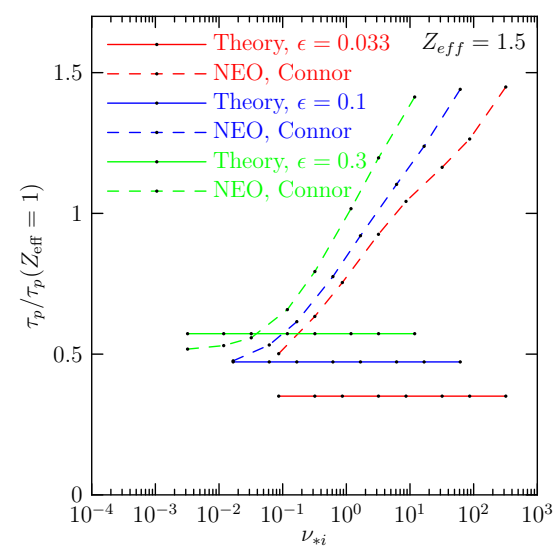

Figure 2: Normalised damping time versus $\nu_{* i}$

to unshifted circular flux surfaces with $R=R_{0}+r \cos \theta$, magnetic field variation $B=B_{0} /\left(1+r \cos \theta / R_{0}\right)$, and constant flux function $I(\psi)=R_{0} B_{0}$. $)$ The electron parameters are assumed to be fixed, with $\tau_{e e}^{-1} /\left(c_{s} / a\right)=10^{-2}$, where $c_{s}=\sqrt{T_{e} / m_{i}}$ and $\tau_{e e}^{-1}=\left(\sqrt{2} \pi e^{4} n_{e} \ln \Lambda\right) /\left(m_{e}^{1 / 2} T_{e}^{3 / 2}\right)$. For given $n_{e}, Z$ and $Z_{\text {eff }}$, the ion and impurity densities are determined from quasi-neutrality and the relative ion and impurity collision frequencies are varied accordingly. To be consistent with the theory, in the NEO calculations we neglect the kinetic electron dynamics, which are small. With NEO, the zonal-flow damping time $\tau_{p}$ has been calculated using various collision operators, namely the Connor operator we used in section 3.1, and the more advanced full Hirshman-Sigmar operator [15]. Unlike the Connor operator, which consists of just a Lorentz scattering operator and simple momentum-restoring term, the full Hirshman-Sigmar operator also includes energy diffusion and models for heating friction effects and for the deceleration effect arising from dynamic friction, which has been shown to be important for modelling the neoclassical transport of multi-ion plasmas [11]. Fig. 1 shows the damping time versus effective charge at $\epsilon=0.3$ for both theory and simulation. In order to emphasise the role of the impurities, the ratio of the damping time to that in a pure plasma has been plotted. It is clearly visible that the use of different collision operators does not have any significant influence on the damping time as the curves hardly deviate from each other. This suggests that the form of the momentum correction term is not playing a large role in the dynamics. Moreover, one finds, for regimes in which the theory is valid, numerical simulation and analytical theory to be in fairly good agreement. The larger discrepancy for larger $Z_{\text {eff }}$ is due to the fact that the effective charge approaches the impurity charge, i.e. violation of the ordering assumptions as the impurities stop being a minority. The agreement improves for smaller $\epsilon$; however, it is not possible to reasonably compare simulation and theory for much smaller $\epsilon$ as the simulation is carried out keeping the collision 

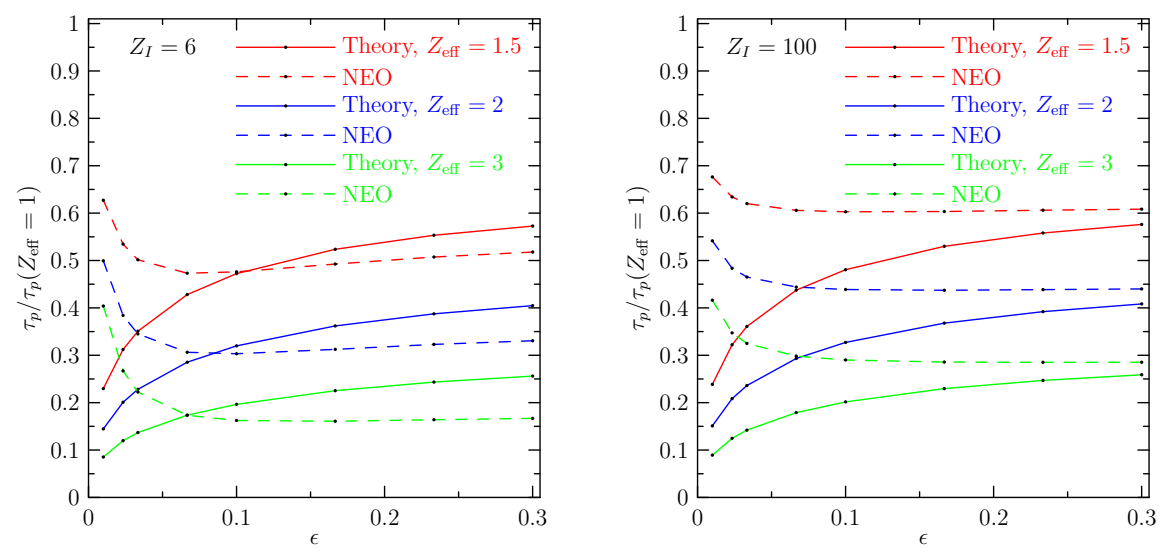

(a) $R_{0} / a=3$
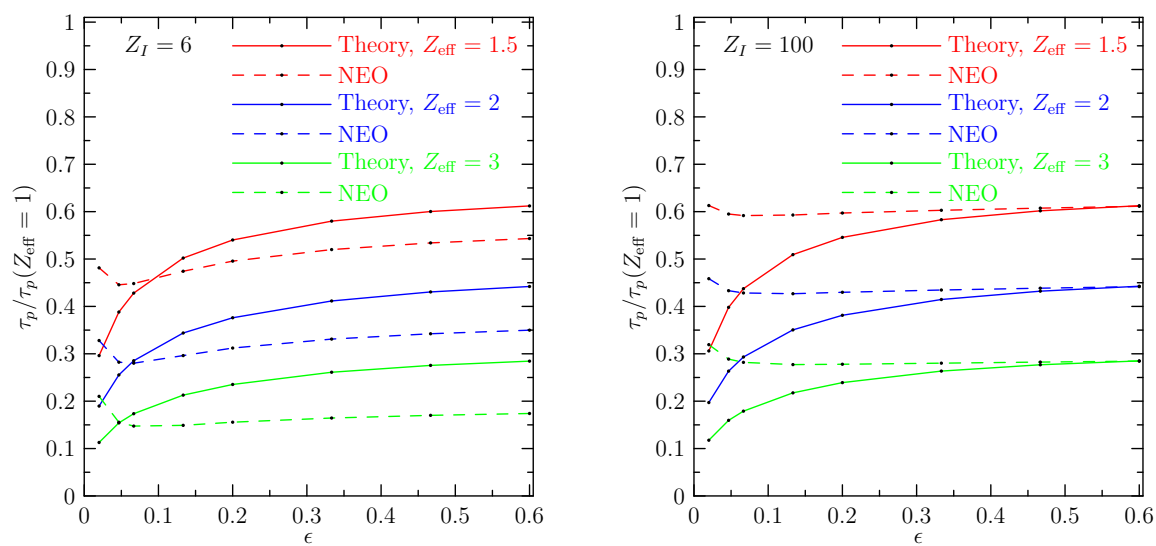

(b) $R_{0} / a=1.5$

Figure 3: Normalised damping time versus $\epsilon$

frequency fixed, which leads to a failure of the banana regime assumption of small effective collision frequency compared with the bounce frequency. In order to further illustrate for which parameters the theory is valid, Fig. 2 shows the dependence of $\tau_{p}$ on $\nu_{* i} \equiv \nu_{i} /\left(\epsilon \omega_{b}\right)$. Obviously, $\epsilon=0.1$ or smaller would require going to much smaller collision frequencies than done here in order to keep the theory valid, which is difficult numerically. In Figs. 3, the damping time has been plotted versus $\epsilon$ for different values of $Z$, where in Fig. 3(b) the aspect ratio has been changed to $R / a_{0}=1.5$ to allow for higher values of $\epsilon$. Clearly visible is the asymptotic behaviour for $Z \rightarrow \infty$, which was assumed in the theory, and again the theory fails for small $\epsilon$ as the plasma leaves the banana regime. 


\section{Discussion}

Before summarising our conclusions in the next section, it is useful to reconsider the validity of the calculation carried out in the two preceding sections and discuss its possible usefulness. In a plasma with electrostatic turbulence, the problem of actual interest is not the initial-value problem (1), but

$$
\frac{\partial f_{a}}{\partial t}+\left(\mathbf{v}_{\|}+\mathbf{v}_{d}+\tilde{\mathbf{v}}_{E}\right) \cdot \nabla f_{a}+\dot{w} \frac{\partial f_{a}}{\partial w}=C_{a}\left(f_{a}\right)
$$

where $\tilde{\mathbf{v}}_{E}=\mathbf{b} \times \nabla \tilde{\phi} / B$ denotes the fluctuating $E \times B$ velocity, averaged over gyromotion. Following Hinton and Rosenbluth [7], we take an average over the toroidal angle to isolate the axisymmetric component, $\bar{f}_{a}$, and obtain

$$
\frac{\partial \bar{f}_{a}}{\partial t}+\left(\mathbf{v}_{\|}+\mathbf{v}_{d}\right) \cdot \nabla \bar{f}_{a}+\dot{w} \frac{\partial \bar{f}_{a}}{\partial w}-C_{a}\left(\bar{f}_{a}\right)=S_{a}
$$

where the source term

$$
S_{a}=-\frac{1}{2 \pi B} \oint(\mathbf{b} \times \nabla \tilde{\phi}) \cdot \nabla f_{a} d \varphi
$$

only has relatively weak contributions from the axisymmetric components of either $\tilde{\phi}$ or $f_{a}$. The point is that the axisymmetric components only contribute through the term

$$
(\mathbf{b} \times \nabla \bar{\phi}) \cdot \nabla \bar{f}_{a}=I\left(\frac{\partial \bar{f}_{a}}{\partial \psi} \nabla_{\|} \bar{\phi}-\frac{\partial \bar{\phi}}{\partial \psi} \nabla_{\|} \bar{f}_{a}\right),
$$

involving parallel gradients, whilst the non-axisymmetric components of $\tilde{\phi}$ and $f_{a}$ also contribute through their perpendicular gradients, which are much larger in gyrokinetic theory. Hinton and Rosenbluth therefore took $S_{a}$ to be given when calculating the axisymmetric components. Since the resulting equation (26) is linear, it can be solved as an initial value problem. If one calculates the response to an initial perturbation, the general solution can be obtained by convoluting the solution of the initial-value problem with the source term $S_{a}$ (see appendix). The latter can be arbitrary, and it does not matter, for instance, whether its auto-correlation time is long or short compared with the collision time.

However, in practice the source term $S_{a}$ is not known and does contain contributions from the axisymmetric part $\bar{f}_{a}$. One might then ask the question whether the turbulent transport may carry particles across a radial wavelength of the zonal flow in a time shorter than the collision time. The zonal flow would then be damped by diffusion caused by the fluctuating $E \times B$ drift (turbulent viscosity) rather than collisions. If the global confinement time is denoted by $\tau_{E}$, then the time required for diffusion across a fraction $\Delta r / a$ of the cross section is

$$
\tau_{D} \sim \tau_{E}\left(\frac{\Delta r}{a}\right)^{2}
$$


and collisional damping is only important for zonal flows with a radial wavelength longer than

$$
\frac{\Delta r}{a}>\left(\frac{\tau_{p}}{\tau_{E}}\right)^{1 / 2},
$$

where $\tau_{p}$ is the neoclassical polarisation time (25) due to collisions that we have calculated. Depending on plasma parameters, this condition may or may not be satisfied - a limitation that seems not to have been pointed out in earlier papers on collisional damping of zonal flows.

It should perhaps be mentioned that there is another application of our results, which has to do with the question of how the plasma responds to a sudden change of the pressure profile. Conventional neoclassical theory predicts the level of poloidal plasma rotation in steady state. In the LH-transition, the pressure gradient changes very rapidly, and one may ask the question of how quickly a new steady state is attained. This is of interest when trying to resolve the "chicken-and-egg" problem of whether sheared rotation causes the H-mode or is merely a consequence of the increased gradients in the pedestal. Since the pressure gradient in $f_{a_{0}}$ enters in exactly the same way as the radial electric field in the kinetic equation (2), this problem is identical to the zonal flow damping problem, and it can be concluded that the new equilibrium is established on the time scale (25).

\section{Conclusions and Summary}

We have considered the effect of heavy, highly charged impurity ions on zonalflow damping in tokamaks. Although such impurities do not affect the first, collisionless stage of the damping, they do accelerate the collisional damping since they increase the effective collisionality of the bulk ions roughly by a factor $Z_{\text {eff }}$. It is possible to calculate the time history of the damping by expanding the pitch-angle dependence of the distribution function in eigenfunctions of the orbit-averaged pitch-angle-scattering operator. However, in the long-time limit, i.e. at late times during the damping process, the problem can be reduced to solving a neoclassical Spitzer problem, and this is also sufficient to calculate the overall damping time defined by Eq. (25).

Collisional damping of zonal flows occurs as a result of friction between the circulating and trapped particles. The latter are locked in the magnetic well on the outboard side of the torus and are therefore unable to rotate poloidally. The circulating ions experience friction against this stationary, trapped population, which damps the rotation. The damping time (25) therefore increases with the effective fraction of circulating particles. In the limit of very tight aspect ratio (the edge region in a spherical tokamak), where the circulating particle fraction is small, $f_{c} \rightarrow 0$, the damping becomes instantaneous, independently of whether impurities are present in the plasma. In the opposite limit of very few trapped particles, $f_{c} \rightarrow 1$, the damping occurs much more quickly if impurity ions are present than otherwise. This has to do with the fact that the time 
it takes for the trapped and passing populations to reach a mutual collisional equilibrium is much shorter for the impurities than for the bulk ions, because of the shorter collision time of the former. Therefore, as soon as the poloidal impurity rotation has been damped, the bulk ions experience friction against the entire impurity population, not just the trapped impurities. This is much more effective than the friction against just the trapped bulk ions when $f_{c}$ is close to unity. Mathematically, this is reflected by the fact that the denominator of Eq. (24) does not vanish in the limit $f_{c} \rightarrow 1$. Physically, it has the effect that the zonal flow damping is enhanced by more than a factor $Z_{\text {eff }}$ when the aspect ratio is large.

These analytical predictions, which are made using a simple pitch-anglescattering collision operator with a momentum-restoring term, seem largely insensitive to the choice of collision operator. Indeed, because the zonal flow damping time can be reduced to a neoclassical Spitzer problem, it can be calculated by neoclassical transport codes, which makes it possible to use still more accurate collision operators and to treat cases of finite collisionality. As long as the particles are in the low-collisionality banana regime, the results are in very good agreement.

It is clear from these results that, when a realistic amount of impurities are present in a tokamak plasma, the collisional damping of zonal flows occurs significantly faster than otherwise. Insofar as this damping mechanism is important, this would suggest that impurities inhibit zonal flows and could have a deleterious effect on confinement.

\section{Appendix: Arbitrary source term $S_{a}$}

The problem (26) with an arbitrary source term amounts to finding the functions $G_{a}$ and $\Phi^{\prime}$, which satisfy

$$
\begin{aligned}
M_{a}\left[\hat{G}_{a}\right] & =\hat{S}_{a} \\
\Phi^{\prime}(t) & =\sum_{a}\left\langle\frac{I}{B} \int m_{a} v_{\|} G_{a} d^{3} v\right\rangle / \sum_{a}\left\langle m_{a} n_{a} R^{2}\right\rangle \\
G_{a}(0) & =0, \quad \Phi^{\prime}(0)=0
\end{aligned}
$$

where the operator $M_{a}$ is defined as

$$
M_{a}[h] \equiv p h+v_{\|} \nabla_{\|} h-\frac{I v_{\|}}{\Omega_{a}} \frac{e_{a}}{T_{a}} f_{a_{0}} p \sum_{a}\left\langle\frac{I}{B} \int m_{a} v_{\|} h d^{3} v\right\rangle / \sum_{a}\left\langle m_{a} n_{a} R^{2}\right\rangle .
$$


We already know the solutions $\hat{g}_{a}$ and $\hat{\phi}^{\prime}$ to

$$
\begin{aligned}
M\left[g_{a}\right] & =k \frac{I m_{a} v_{\|}}{B} f_{a_{0}}=L_{a}\left[g_{a}\right] \\
\phi^{\prime}(t) & =\sum_{a}\left\langle\frac{m_{a} n_{a}|\nabla \psi|^{2}}{B^{2}} \phi^{\prime}(0)+\frac{I}{B} \int m_{a} v_{\|} g_{a} d^{3} v\right\rangle / \sum_{a}\left\langle m_{a} n_{a} R^{2}\right\rangle \\
g_{a}(0) & =\frac{I v_{\|}}{\Omega_{a}} \frac{e_{a}}{T_{a}} \phi^{\prime}(0) f_{a_{0}}, \quad \phi^{\prime}(0)=\phi_{0}^{\prime},
\end{aligned}
$$

where

$$
L_{a}[h] \equiv p h-v_{\|} \nabla_{\|} h-\frac{I v_{\|}}{\Omega_{a}} \frac{e_{a}}{T_{a}} f_{a_{0}} p \sum_{a}\left\langle\frac{I}{B} \int m_{a} v_{\|} h d^{3} v\right\rangle / \sum_{a}\left\langle m_{a} n_{a} R^{2}\right\rangle
$$

only differs from $M_{a}$ in the reversed sign in front of the term containing the parallel gradient. As we found $g_{a}$ to be odd in $\sigma$, it is also a solution to the problem including the operator $L_{a}$. When all ion temperatures are equilibrated, the constant $k \equiv \frac{1}{T_{a}} \sum_{a}\left\langle\frac{m_{a} n_{a}|\nabla \psi|^{2}}{B^{2}}\right\rangle \phi^{\prime}(0) / \sum_{a}\left\langle m_{a} n_{a} R^{2}\right\rangle$ is independent of the particle species.

Since, for the unlike-species collision operator, the relation

$$
\sum_{a, b} \int d^{3} v \frac{g_{a}}{f_{a_{0}}} C_{a b}\left(f_{a}, f_{b}\right)=\sum_{a, b} \int d^{3} v \frac{f_{a}}{f_{a_{0}}} C_{a b}\left(g_{a}, g_{b}\right)
$$

holds for any pair of distributions $g_{a}, f_{a}$ [16], the operators $M_{a}$ and $L_{a}$ are adjoint in sense that

$$
\sum_{a}\left\langle\int \frac{\hat{G}_{a}}{f_{a_{0}}} L_{a}\left[\hat{g}_{a}\right] d^{3} v\right\rangle=\sum_{a}\left\langle\int \frac{\hat{g}_{a}}{f_{a_{0}}} M_{a}\left[\hat{G}_{a}\right] d^{3} v\right\rangle .
$$

Thus, to calculate the potential response (27), one can use

$$
\begin{aligned}
\sum_{a}\left\langle\frac{I}{B} \int m_{a} v_{\|} \hat{G}_{a} d^{3} v\right\rangle & =\frac{1}{k} \sum_{a}\left\langle\int \frac{\hat{G}_{a}}{f_{a_{0}}} L_{a}\left[\hat{g}_{a}\right] d^{3} v\right\rangle \\
& =\frac{1}{k} \sum_{a}\left\langle\int \frac{\hat{g}_{a}}{f_{a_{0}}} M_{a}\left[\hat{g}_{a}\right] d^{3} v\right\rangle \\
& =\frac{1}{k} \sum_{a}\left\langle\int \frac{\hat{g}_{a} \hat{S}_{a}}{f_{a_{0}}} d^{3} v\right\rangle .
\end{aligned}
$$

As mentioned in Sec. 4 and in [7], the response to a source is thus obtained by convoluting this source with the solution of the initial value problem.

\section{References}

[1] G.W. Hammett, M.A. Beer, W. Dorland, S.C. Cowley, and S.A. Smith. Developments in the gyrofluid approach to Tokamak turbulence simulations. Plasma Phys. Control. Fusion, 35:973-985, 1993. 
[2] R.E. Waltz, G.D. Kerbel, and J. Milovich. Toroidal gyro-Landau fluid model simulations in a nonlinear ballooning mode representation with radial modes. Physics of Plasmas, 1:2229-2244, 1994.

[3] A.M. Dimits, T.J. Williams, J.A. Byers, and B.I. Cohen. Scalings of IonTemperature-Gradient-Driven Anomalous Transport in Tokamaks. Phys. Review Letters, 77:71-74, 1996.

[4] Z. Lin, T.S. Hahm, W.W. Lee, W.M. Tang, and R.B. White. Gyrokinetic simulations in general geometry and applications to collisional damping of zonal flows. Physics of Plasmas, 7:5, 2000.

[5] P.H. Diamond, S-I. Itoh, K. Itoh, and T.S. Hahm. Zonal flows in plasma a review. Plasma Phys. Control. Fusion, 47:R35-R161, 2005.

[6] R.C. Morris, M.G. Haines, and R.J. Hastie. The neoclassical theory of poloidal flow damping in a tokamak. Physics of Plasmas, 3:4513, 1996.

[7] F.L. Hinton and M.N. Rosenbluth. Dynamics of axisymmetric $(\mathrm{E} \times \mathrm{B})$ and poloidal flows in tokamaks. Plasma Phys. Control. Fusion, 41:A653-A662, 1999.

[8] Y. Xiao, P.J. Catto, and K. Molvig. Collisional damping for ion temperature gradient mode driven zonal flow. Physics of Plasmas, 14:032302, 2007.

[9] M.N. Rosenbluth and F.L. Hinton. Poloidal Flow Driven by IonTemperature-Gradient Turbulence in Tokamaks. Phys. Rev. Lett., 80:724$727,1998$.

[10] C.T. Hsu, P.J. Catto, and D.J. Sigmar. Neoclassical transport of isotropic fast ions. Physics of Fluids B2, 2:0899-8221/90/020280-11, 1990.

[11] E.A. Belli and J. Candy. Kinetic calculation of neoclassical transport including self-consistent electron and impurity dynamics. Plasma Phys. Control. Fusion, 50:095010, 2008.

[12] P. Helander and D. J. Sigmar. Collisional Transport in Magnetized Plasmas. Cambridge University Press, 2002.

[13] J.W. Connor. The neo-classical transport theory of a plasma with multiple ion species. Plasma Physics, 15:765, 1973.

[14] S.P. Hirshman and D.J. Sigmar. Neoclassical transport of impurities in tokamak plasmas. Nucl. Fusion, 21:1079, 1981.

[15] S.P. Hirshman and D.J. Sigmar. Approximate Fokker-Planck collision operator for transport theory applications. Phys. Fluids, 19:1532-1540, 1976.

[16] F.L. Hinton. Collisional transport in plasma. In Basic Plasma Physics, volume 1. North-Holland Publishing Company, 1983. 